\title{
Correction to: A Comparative Analysis of Machine Enep Learning Algorithms for Intrusion Entection in WSN
}

Saurabh Deshpande, J. Gujarathi, P. Chandre, and Pravin Nerkar

\section{Correction to:}

Chapter "A Comparative Analysis of Machine Deep

Learning Algorithmsfor Intrusion Detection in WSN" in:

P. N. Mahalle et al. (eds.),

Security Issues and Privacy Threats in Smart Ubiquitous

Computing, Studies in Systems, Decision and Control 341, https://doi.org/10.1007/978-981-33-4996-4_11

The original version of the book was published with incorrect author name, "Shruti Deshpande" has as "Saurabh Deshpande" in the chapter "A Comparative Analysis of Machine Deep Learning Algorithms for Intrusion Detection in WSN". The chapter and book have been updated with change. 